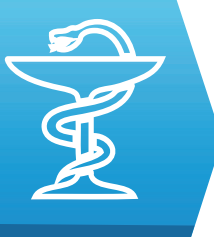

PHARMACY

1) Department of Pharmaceutical Technology and Biopharmacy, Faculty of Pharmacy, Iuliu Hatieganu University of Medicine and Pharmacy, Cluj-Napoca, Romania

2) Department of Toxicology, Faculty of Pharmacy, Iuliu Hatieganu University of Medicine and Pharmacy, Cluj-Napoca, Romania

DOI: $10.15386 / \mathrm{mpr}-1225$

Manuscript received: 02.11.2018

Received in revised form: 17.01.2019

Accepted: 27.01.2019

Address for correspondence: maria.abrudan@umfcluj.ro

\title{
Pharmacokinetic interactions study between carvedilol and some antidepressants in rat liver microsomes - a comparative study
}

Maria Bianca Abrudan¹, Daniela Saveta Popa ${ }^{2}$, Dana Maria Muntean¹, Ana Maria Gheldiu ${ }^{1}$, Laurian Vlase ${ }^{1}$

\begin{abstract}
Background and aims. Cardiovascular diseases and depressive disorders are some of the most frequent diseases. The probability of concomitant prescription of antihypertensive and antidepressive medication is increasing. The aim of this study was to investigate the enzyme inhibition by bupropion, sertraline and fluvoxamine on the metabolism of carvedilol using rat pooled liver microsomes and to assess the importance of these interactions from the pharmacokinetic mechanism point of view.

Methods. Two substrate concentrations $(0.5$ and $1 \mu \mathrm{M})$ and four inhibitor concentrations $(0,0.1,0.75$ and $1.5 \mu \mathrm{M})$ were used for each tested inhibitor.

Results. The results of the in vitro experiments showed a significant decrease of the metabolic rate of carvedilol to 4'-hydroxyphenyl carvedilol, for all tested inhibitors, when the inhibitor was added to the incubation mixture containing the substrate. Moreover, an increase of the area under the concentration-time curve for carvedilol was observed after incubation with each tested inhibitor compared with the control state (no inhibitor). The most potent inhibitor was sertraline, followed by fluvoxamine and bupropion.
\end{abstract}

Conclusion. The co-administration of tested antidepressants led to a significant alteration of carvedilol's metabolism in vitro. CYP2D6 inhibition is the main pharmacokinetic mechanism that can explain these drug-drug interactions, with possible clinical implications.

Keywords: pharmacokinetics, CYP2D6, rat liver microsomes, carvedilol, antidepressants

\section{Background and aims}

Cardiovascular diseases are the first cause of death globally: more people die annually from cardiovascular diseases than from any other cause. An estimated 17.7 million people died from cardiovascular diseases in 2015 , representing $31 \%$ of all global deaths [1]. Carvedilol is a third generation vasodilating beta-blocker with antioxidant activities currently marketed for the treatment of hypertension and congestive heart failure [2]. Having a nonselective beta-adrenoceptor blocking activity, carvedilol blocks both beta-1 and alpha-1 adrenergic receptors; it has a higher affinity to beta- 1 receptors than alpha- 1 receptors [3]. Carvedilol is administered as a racemic mixture, both $\mathrm{S}$ and $\mathrm{R}$ enantiomers have equal alpha blocking effects but $\mathrm{S}$ enantiomer shows stronger beta blockade [4].
A highly lipophilic drug, carvedilol is rapidly absorbed and is metabolized mainly by the liver via cytochrome P450 (CYP) enzymes [5]. The most important isoenzyme involved in carvedilol's metabolism is CYP2D6, which leads to the formation of 5'-hydroxyphenyl carvedilol and 4'-hydroxyphenyl carvedilol. To a lesser extent, isoenzyme CYP2C9 is involved in converting carvedilol to O-desmethyl carvedilol and isoenzyme CYP1A2 leads to formation of 8-hydroxy carbazolyl carvedilol [6]. From these metabolites, only the metabolite 4'-hydroxyphenyl carvedilol may contribute to the beta-blocking activity of carvedilol, due to the fact that it possesses approximately 13 times more potency than carvedilol itself [7].

Depression is a widespread disorder nowadays, when more than 300 million 
people are affected all over the world [8]. Antidepressive drugs, especially those from the group of selective serotonin reuptake inhibitors (SSRIs) and serotonin-noradrenaline reuptake inhibitors (SNRIs), are frequently prescribed to the patients affected by depressive disorders. The probability that cardiac patients also suffer from depression is increasing, as well as the probability of concomitant prescription for antihypertensives and antidepressants. Considering this clinical context, we considered useful to evaluate the interactions that can occur between carvedilol and frequently prescribed antidepressants: bupropion, sertraline and fluvoxamine.

Bupropion is a dual norepinephrine and dopamine reuptake inhibitor used as antidepressant medication to treat major depressive disorder or seasonal affective disorder [9]. It can also be used to help people stop smoking by reducing cravings and other withdrawal effects, or as treatment of bipolar disorder and attention-deficit hyperactivity disorder $[10,11]$. Bupropion inhibits CYP2D6 activity as shown in vitro [12] and in vivo studies [13].

Sertraline and fluvoxamine are selective serotonin reuptake inhibitors used as antidepressant or antianxiety agents in disorders like major depression, panic disorder, obsessive-compulsive disorder, or social phobia $[14,15]$. These second-generation antidepressants are among the most prescribed drugs in depression [16], having a high probability to be involved in drug interactions due to their inhibitory potential upon CYP isoenzymes. Sertraline is a moderate inhibitor of CYP2D6 and a weak inhibitor of CYP1A2 and CYP2C9, whereas fluvoxamine is a weak inhibitor of CYP2D6, a moderate inhibitor of CYP2C9 and a strong inhibitor of CYP1A2 [17]. All of them inhibit in a different manner the main isoenzyme involved in carvedilol's metabolism (CYP2D6).

Due to carvedilol's extensive oxidative metabolism in the liver, drugs that induce or inhibit carvedilol oxidation can affect its pharmacokinetics (PKs). Therefore, concomitant administration of drugs that act as inhibitors like bupropion, sertraline and fluvoxamine may influence its elimination, leading to changes in plasma concentrations and subsequent clinical effects. However, the effects of bupropion, sertraline and fluvoxamine on the PKs of carvedilol in vitro have not been reported.

Thus, the aim of this study was to investigate in vitro PK interactions that occur between carvedilol and bupropion / sertraline / fluvoxamine and to assess their inhibitory magnitude. The results are important to predict the interaction potential between these drugs, to describe the mechanism of these PK interactions, to characterize the safety profile and the pharmacotherapy of carvedilol.

\section{Methods}

\section{Chemicals and reagents}

Carvedilol, bupropion, sertraline and fluvoxamine were purchased from Moehs (Rubí, Spain). The metabolite 4'-hydroxyphenyl carvedilol was bought from Toronto Research Chemicals (Toronto, Ontario, Canada). HPLCgrade acetonitrile, $98 \%$ formic acid and methanol of analytical-reagent grade were purchased from Merck KGaA (Darmstadt, Germany). Heparine sodique 25000 $\mathrm{UI} / 5 \mathrm{~mL}(5000 \mathrm{UI} / \mathrm{mL})$ was acquired from Panpharma Laboratoires (France). Tris, glycerol, potassium chloride, EDTA, magnesium chloride, $\beta$-nicotinamide adenine dinucleotide phosphate (NADP), glucose 6-phosphate (G6P), glucose 6-phosphate dehydrogenase (G6PDH), bovine serum albumin (BSA) were purchased from SigmaAldrich (St. Louis, Missouri, USA).

The equipment used in this study were: an Agilent 1100 series - HPLC system (consisting of binary pump, autosampler, thermostat) (Agilent Technologies, USA), coupled with a Fluorescence detector, an Agilent 1100 series - HPLC system (consisting of binary pump, autosampler, thermostat) (Agilent Technologies, USA), coupled with a Bruker Ion Trap VL (Bruker Daltonics GmbH, Germany) and a Sorvall WX 100 ultracentrifuge (Thermo Fisher Scientific, USA).

\section{Preparation of rat liver microsomes}

White male Charles River Wistar rats Crl:WI $(\mathrm{n}=4)$ weighing 270 to $390 \mathrm{~g}$ were purchased from the Experimental Medicine Centre and Practical Skills (ClujNapoca, Romania). The working protocols were reviewed and approved by the Ethics Committee of the Iuliu Hatieganu University of Medicine and Pharmacy, ClujNapoca, Romania (no. 531/23.12.2015). The study was conducted in accordance with the specific regulations and amendments: the 'Guiding Principles in the Use of Animals in Toxicology' adopted by the Society of Toxicology (USA) and the U.K. Animals (Scientific Procedures) Act, 1986 and associated guidelines, EU Directive 2010/63/EU for animal experiments.

Rat pooled liver microsomes were isolated by differential centrifugation, using an adapted method previously described by Kremers et al. [18,19]. Shortly, the anesthesia was induced by an intramuscular injection with a $1 \mathrm{ml} / \mathrm{kg}$ dose of ketamine/xylazine/diazepam 1:1:1 $(\mathrm{V} / \mathrm{V} / \mathrm{V})(100 \mathrm{mg} / \mathrm{mL}, 20 \mathrm{mg} / \mathrm{mL}, 5 \mathrm{mg} / \mathrm{mL})$ before surgery and was followed by a dose of anticoagulant administered intravenously (i.v.) (heparin 500U/kg). Then, all anatomic layers were cut and the abdomen was opened, thus allowing direct access to the liver. An i.v. cannula $20 \mathrm{G}$ (Pink) $1.1 \mathrm{x}$ 32mm, flow: $62 \mathrm{ml} / \mathrm{min}$ (Med Devices Lifesciences Ltd., London, UK) inserted in the portal vein was used to perfuse the liver with an iced-cold wash buffer containing $50 \mathrm{mM}$ Tris (pH 7.4) and $100 \mathrm{mM} \mathrm{KCl} \mathrm{(Buffer} \mathrm{1),} \mathrm{at} \mathrm{the} \mathrm{same} \mathrm{time}$ an incision was performed in the subclavian vein. At the end of the perfusion, the liver was excised with scissors. The liver tissue was broken down in $110 \mathrm{~mL}$ ice-cold wash buffer (Buffer 1) in a potter homogenizer by gentle tapping with a stirring rod, followed by an ultrasound homogenization for 3 minutes. The hepatocytes homogenate thus obtained was then 
centrifuged at $13000 \mathrm{xg}$ for 30 minutes, at $4^{\circ} \mathrm{C}$. The resulting pellets were resuspended in a fresh solution of wash buffer, being again centrifuged at $13000 \mathrm{xg}$ for 30 minutes, at $4^{\circ} \mathrm{C}$. Then, all the supernatants were carefully removed and added to the first supernatant fraction. All the reunited fractions were then centrifuged at $105000 \mathrm{xg}$ for 60 minutes, at $4^{\circ} \mathrm{C}$, to obtain the microsomal pellets. Pellets were resuspended in another buffer (Buffer 2) containing $100 \mathrm{mM}$ Tris (pH 7.4), $1 \mathrm{mM}$ EDTA and 20\% glycerol (v/v). Microsomal fractions were aliquoted and stored at $-80^{\circ} \mathrm{C}$ until the experiments.

The microsomal protein concentration was determined by the Bradford method using bovine serum albumin (BSA) as standard.

\section{Incubation procedure}

The microsomal incubation procedure used was similar to that described previously [20,21]. Incubations were performed at $37^{\circ} \mathrm{C}$ with 0.5 and $1 \mu \mathrm{M}$ carvedilol and $0,0.1,0.75$ or $1.5 \mu \mathrm{M}$ bupropion, sertraline or fluvoxamine. Incubation mixtures contained $0.25 \mathrm{mg}$ microsomal protein/ $\mathrm{mL}$, one antidepressant as enzymatic inhibitor, a NADPH regenerating system $(1 \mathrm{mM}$ NADP, $5 \mathrm{mM}$ G6P, $1 \mathrm{U} / \mathrm{mL}$ G6PDH, $5 \mathrm{mM} \mathrm{MgCl}_{2}$ ), the substrate (carvedilol) and $5 \mathrm{mM}$ Tris buffer ( $\mathrm{pH}$ 7.4). Before starting the reaction by adding the NADPH regenerating system, there was a 15-minute preincubation period at $37^{\circ} \mathrm{C}$ in a shaking water bath. The reactions were stopped by adding $100 \mu \mathrm{L}$ of cold acetonitrile to $100 \mu \mathrm{L}$ microsome mixture and cooling on ice, at different time points $(0.5,1,2,3,5,7,10,15,20,25$ and 30 minutes). After being vortexed for 10 seconds, the samples were centrifuged at $12000 \mathrm{rpm}, 13201 \mathrm{xg}$ for 5 minutes. Then, 5 $\mu \mathrm{L}$ of the supernatants were subjected to high-performance liquid chromatography with fluorescence detection (HPLCFLD) assay. For sertraline, the detection was performed by high-performance liquid chromatography-mass spectrometry method (HPLC-MS). Using appropriate calibration samples containing known amounts of carvedilol and 4'-hydroxyphenyl carvedilol, concentrations in reaction mixtures were thereby determined. The incubations were completed in triplicate, and the data were expressed as the mean $\pm \mathrm{SD}$ of three experiments.

\section{High-performance liquid chromatography assay}

Concentrations of carvedilol and 4'-hydroxyphenyl carvedilol, for the inhibitors bupropion and fluvoxamine, were determined by HPLC with fluorescence detection (Agilent Technologies, USA). A Zorbax SB-C18 (50 x $2.1 \mathrm{~mm}, 3.5 \mu \mathrm{m}$ ) chromatographic column (Agilent Technologies, USA) was used for separation. The mobile phase consisted of a 30:70 (V/V) mixture of acetonitrile and $0.1 \%(\mathrm{~V} / \mathrm{V})$ phosphoric acid in water; the flow rate was $1 \mathrm{~mL} /$ min; the thermostat temperature was set at $45^{\circ} \mathrm{C}$. The peaks were detected by using an Agilent 1100 series fluorescence detector (excitation wavelength $243 \mathrm{~nm}$ and emission wavelength $358 \mathrm{~nm}$ ). The calibration curve was linear over the concentration range $0.02-2.01 \mu \mathrm{M}$ for carvedilol and $0.02-2.04 \mu \mathrm{M}$ for its metabolite, 4'-hydroxyphenyl carvedilol. The method showed a good linearity ( $r>0.992)$, intra- and inter-day precision $(\mathrm{CV} \%<10.8 \%)$ and accuracy (bias $<10.1 \%$ ) over the range of calibration curve; the overall recovery was between $97.5-114.9 \%$.

When sertraline was the inhibitor used in incubations, carvedilol concentrations were determined by a validated liquid chromatography-mass spectrometry method previously reported [22]. The HPLC system was an Agilent 1100 series (binary pump, autosampler, thermostat) (Agilent Technologies, USA) and was coupled with a Bruker Ion Trap VL (Bruker Daltonics GmbH, Germany). Briefly, a Zorbax SB-C18 chromatographic column (50 x $2.1 \mathrm{~mm}, 3.5 \mu \mathrm{m}$ ) (Agilent Technologies, USA) was used. The mobile phase consisted of a $34: 66(\mathrm{~V} / \mathrm{V})$ mixture of acetonitrile and $0.2 \%(\mathrm{~V} / \mathrm{V})$ formic acid in water. The flow rate was $0.5 \mathrm{~mL} / \mathrm{min}$, and the thermostat temperature was set at $42^{\circ} \mathrm{C}$. The mass spectrometry detection was in multiple reaction monitoring (MRM) mode, positive ionization, using an electrospray ionization source. The ion transitions monitored were $\mathrm{m} / \mathrm{z} 222,224,283$ from $\mathrm{m} / \mathrm{z}$ 407 for carvedilol (MRM mode). The calibration curve of carvedilol was linear at a concentration range of 0.09-1.19 $\mu \mathrm{M}$, with a correlation coefficient of 0.9995 .

\section{Kinetic and statistical analysis}

The parameters: metabolization rate (nmol/ $\mathrm{min} / \mathrm{mg}$ protein), area under the concentration-time curve $\left(\mathrm{AUC}_{0-30}, \mathrm{~min} * \mathrm{nmol} / \mathrm{mL}\right)$, the percentage $(\%)$ of metabolized carvedilol and the percentage (\%) of resulted metabolite, 4'-hydroxyphenyl carvedilol, were calculated for all in vitro experiments, after 30 minutes of incubation in rat pooled liver microsomes systems. All kinetic data were expressed as the mean \pm standard deviation (SD) of 3 microsomal experiments. The statistical analyses were performed by one-way analysis of variance (ANOVA) for intergroup comparison, for each substrate concentration. All analyses had a set level of significance at $p<0.05$.

\section{Results}

The effects of three enzymatic inhibitors on the metabolism of carvedilol were investigated using rat pooled liver microsome incubation systems. Two substrate concentrations $(0.5$ and $1 \mu \mathrm{M})$ and four inhibitor concentrations $(0,0.1,0.75$ and $1.5 \mu \mathrm{M})$ were used for each antidepressant tested as inhibitor. All the incubations were completed in triplicate.

Carvedilol's in vitro metabolism was modified in the presence of analyzed enzymatic inhibitors: sertraline, fluvoxamine and bupropion (Table I). As the concentration of inhibitor increased, the percentage of carvedilol metabolized decreased.

In vitro, carvedilol metabolization rate, calculated after 30 minutes of incubation in rat pooled liver microsomes, was decreased by all tested antidepressants as shown in Figure 1. All the differences were statistically significant $(\mathrm{p}<0.05)$ compared with the control group. 
Table I. The effects of three evaluated antidepressants on carvedilol's metabolism after 30 minutes of incubation in rat liver microsomes (data presented as mean $\pm \mathrm{SD}, \mathrm{n}=3$ ).

\begin{tabular}{|c|c|c|c|c|}
\hline \multirow{3}{*}{$\begin{array}{l}\text { Inhibitor concentration } \\
\qquad(\mu \mathrm{M})\end{array}$} & \multicolumn{4}{|c|}{ Substrate concentration $(\mu \mathrm{M})$} \\
\hline & \multicolumn{2}{|c|}{0.5} & \multicolumn{2}{|l|}{8} \\
\hline & CVD* & 4'OH-CVD** & CVD* & 4'OH-CVD** \\
\hline \multicolumn{5}{|c|}{ Sertraline\# } \\
\hline $\mathbf{0}$ & $99.1 \pm 0.6$ & - & $95.2 \pm 2.1$ & - \\
\hline 0.1 & $39.3 \pm 13.3$ & - & $79.4 \pm 5.4$ & - \\
\hline 0.75 & $32.6 \pm 7.6$ & - & $39.9 \pm 6.4$ & - \\
\hline 1.5 & $55.7 \pm 3.5$ & - & $34.6 \pm 2.6$ & - \\
\hline $\mathrm{p} \bullet$ value (ANOVA) & $0.00002, \mathrm{~S}$ & - & $0.0001, S$ & - \\
\hline \multicolumn{5}{|c|}{ Fluvoxamine } \\
\hline $\mathbf{0}$ & $99.1 \pm 0.6$ & $23.8 \pm 3.7$ & $94.5 \pm 4.2$ & $16.8 \pm 1.2$ \\
\hline 0.1 & $90.4 \pm 1.3$ & $17.6 \pm 0.4$ & $85.1 \pm 2.0$ & $13.7 \pm 1.6$ \\
\hline 0.75 & $78.5 \pm 4.2$ & $14.9 \pm 1.8$ & $74.6 \pm 2.3$ & $10.4 \pm 0.4$ \\
\hline 1.5 & $71.7 \pm 0.4$ & $12.6 \pm 0.6$ & $70.6 \pm 0.7$ & $9.7 \pm 0.4$ \\
\hline p• value (ANOVA) & $0.00001, \mathrm{~S}$ & $0.0024, \mathrm{~S}$ & $0.00001, \mathrm{~S}$ & $0.0001, \mathrm{~S}$ \\
\hline \multicolumn{5}{|c|}{ Bupropion } \\
\hline $\mathbf{0}$ & $99.1 \pm 0.6$ & $23.8 \pm 3.7$ & $94.5 \pm 4.2$ & $16.8 \pm 1.2$ \\
\hline 0.1 & $97.4 \pm 0.3$ & $19.5 \pm 0.5$ & $92.6 \pm 3.9$ & $17.5 \pm 1.8$ \\
\hline 0.75 & $80.8 \pm 6.9$ & $16.2 \pm 2.0$ & $96.3 \pm 0.6$ & $13.4 \pm 0.2$ \\
\hline 1.5 & $73.0 \pm 0.6$ & $11.8 \pm 0.5$ & $80.7 \pm 4.7$ & $12.4 \pm 1.4$ \\
\hline p• value (ANOVA) & $0.00003, S$ & $0.0016, \mathrm{~S}$ & $0.0072, S$ & $0.0034, \mathrm{~S}$ \\
\hline
\end{tabular}

- ANOVA for intergroup comparison, for each substrate concentration, statistically significant (S) when $\mathrm{p}<$ $0.05,{ }^{*} \mathrm{CVD}$ - carvedilol; **4'OH-CVD - 4'-hydroxyphenyl carvedilol; \#for the experiments with sertraline as enzymatic inhibitor, the metabolite quantification was not possible, due to a different detection system.
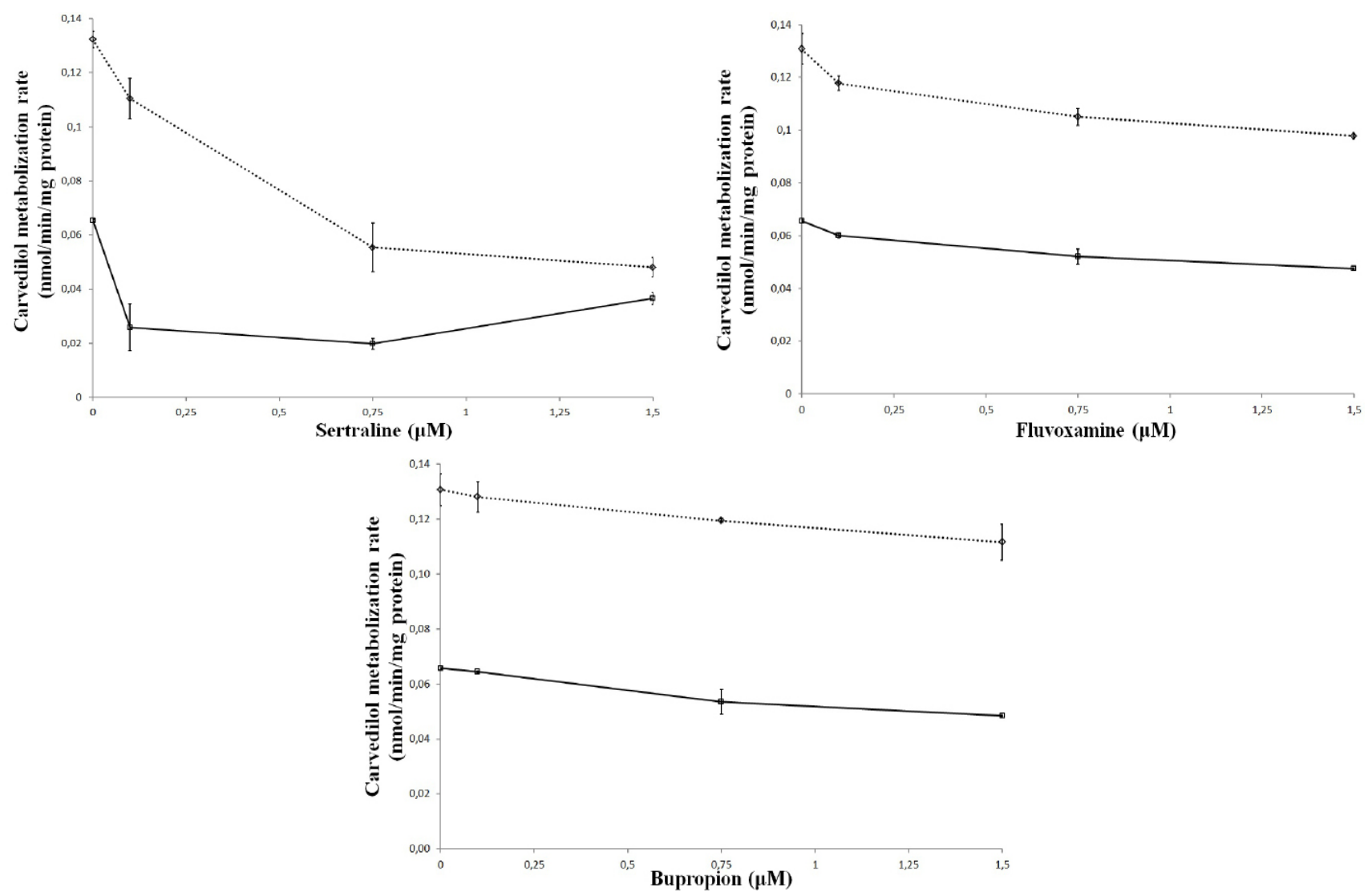

Figure 1. Rate of metabolism (nmol/min/mg protein) of carvedilol in relation to the concentration of antidepressants tested (sertraline, fluvoxamine and bupropion) in rat liver microsomes. Incubations were performed for 30 minutes in the control state (no inhibitor), and with varying concentrations of inhibitors for $0.5(-\diamond \rightarrow)$ and $1 \mu \mathrm{M}$ carvedilol (- - - -). 
Table II. $\mathrm{AUC}_{0-30}(\mathrm{~min} * \mathrm{nmol} / \mathrm{mL})$ of carvedilol in relation to inhibitor's concentration, for each tested antidepressant (sertraline, fluvoxamine and bupropion) in rat liver microsomes. Incubations were performed in the control state (no inhibitor), and with varying inhibitors concentrations for 0.5 and $1 \mu \mathrm{M}$ carvedilol (data presented as mean $\pm \mathrm{SD}, \mathrm{n}=3$ ).

\begin{tabular}{|c|c|c|c|c|}
\hline $\begin{array}{c}\text { Substrate } \\
\text { concentration }(\mu \mathrm{M})\end{array}$ & $\begin{array}{c}\text { Inhibitor } \\
\text { concentration }(\mu \mathrm{M})\end{array}$ & Sertraline & Fluvoxamine & Bupropion \\
\hline \multirow{4}{*}{$1 \mu \mathrm{M}$ CVD } & 0 & $8.2 \pm 0.8$ & $8.1 \pm 1.3$ & $8.1 \pm 1.3$ \\
\hline & 0.1 & $14.9 \pm 2.2$ & $11.2 \pm 0.4$ & $9.8 \pm 1.7$ \\
\hline & 0.75 & $24.0 \pm 1.5$ & $14.4 \pm 0.6$ & $11.1 \pm 0.1$ \\
\hline & 1.5 & $23.5 \pm 0.2$ & $15.7 \pm 0.2$ & $12.7 \pm 1.3$ \\
\hline \multicolumn{2}{|c|}{$p^{*}$ value (ANOVA) } & $<0.05, \mathrm{~S}$ & $0.00001, \mathrm{~S}$ & $0.012, \mathrm{~S}$ \\
\hline \multirow{4}{*}{$0.5 \mu \mathrm{M}$ CVD } & 0 & $2.4 \pm 0.1$ & $2.2 \pm 0.1$ & $2.2 \pm 0.1$ \\
\hline & 0.1 & $10.7 \pm 1.6$ & $4.6 \pm 0.3$ & $3.3 \pm 0.1$ \\
\hline & 0.75 & $11.6 \pm 1.2$ & $6.5 \pm 0.2$ & $6.6 \pm 0.9$ \\
\hline & 1.5 & $9.6 \pm 0.6$ & $7.9 \pm 0.2$ & $7.2 \pm 0.05$ \\
\hline \multicolumn{2}{|c|}{$p^{*}$ value (ANOVA) } & $0.0008, \mathrm{~S}$ & $<0.05, \mathrm{~S}$ & $0.00001, S$ \\
\hline
\end{tabular}

Table II shows the levels of carvedilol (expressed as $\left.\mathrm{AUC}_{0-30}\right)$ in rat pooled liver microsomes after 30 minutes of incubation at 2 substrate concentrations, in the presence and absence of the 3 tested inhibitors (various concentrations). The mean values were determined for all the combinations substrate-inhibitor. The AUC increased as the concentration of inhibitor increased. All the differences were statistically significant $(\mathrm{p}<0.05)$ compared with the control group.

The inhibitor potency could be associated with the extent to which the substrate was altered. The influences on carvedilol's metabolism could be evaluated using any of the above parameters. A decrease in the percentage of metabolized carvedilol was similar to the decrease of metabolization rate and, in the same time, to the $\mathrm{AUC}_{0-30}$ augmentation, consecutive to increasing inhibitor concentrations. From the three tested inhibitors, despite the substrate concentration, sertraline was proved to be the most potent inhibitor. For instance, at the highest evaluated inhibitor and substrate concentration, the metabolisation rate decreased with $63.63 \%$ for sertraline, with $25.19 \%$ for fluvoxamine and with $14.5 \%$ for bupropion. For the other 2 inhibitors (fluvoxamine and bupropion), their potencies were relatively close at small substrate concentrations, but at higher substrate concentrations it seemed that fluvoxamine was a more potent inhibitor than bupropion.

\section{Discussion}

Cardiovascular disease is the leading cause of death, disability, and disease burden in the developed world. Depression is common in cardiovascular disease patients, being linked to higher mortality and morbidity rates [12]. For this reason, drugs used to treat cardiovascular diseases and depression, are likely to be administered together in therapy.

Many drug interactions occur due to an altered CYP450 metabolism. These drugs that affect one substance metabolism can be either inhibitors or inducers. Inhibitors block the metabolic activity of one or more CYP450 enzymes. The magnitude to which an inhibitor affects a drug metabolism can be variable upon factors such as the dose or the inhibitor's ability to bind to the enzyme [23].

In vitro metabolic models using liver microsomes can be applied to predict drug interactions caused by reversible inhibition of metabolism [24]. Understanding and anticipating drug interactions is a necessary part of rational therapeutics, especially when the efficacy or toxicity of a medication is changed by the administration of another compound [25].

Firstly, carvedilol is mainly metabolized by the liver via CYP2D6 isoenzyme and to a lower extent, by CYP2C9 and CYP1A2 [5,6]. Secondly, sertraline, fluvoxamine and bupropion are inhibitors of these metabolic pathways [17]. Considering the above mentions in addition to nowadays situation, when mortality and morbidity rates are increasing in patients suffering from depression associated with a cardiovascular disease [12], it is important to study these metabolic drug-drug interactions. Inhibitors of CYP2D6 activity, such as quinidine, paroxetine, fluoxetine or propafenone may increase plasmatic concentrations of carvedilol. Therefore, patients taking these drugs might be at risk of hypotension due to an excessive $\alpha$ - and $\beta$ adrenoreceptor blockade [26].

The results of these in vitro experiments demonstrated drug-drug interactions between carvedilol and three antidepressant drugs that are CYP2D6 enzyme inhibitors (sertraline, fluvoxamine, bupropion). The existence of enzymatic inhibition is proved by statistically significant $(p<0.05)$ alteration of parameters like metabolization rate and area under the concentration-time curve, compared with the control group (substrate without inhibitor). In the presence of inhibitor, the metabolism of carvedilol was modified. The effects of sertraline, fluvoxamine and bupropion on in vitro carvedilol's metabolism are due to enzymatic inhibition of the main isoenzyme responsible for carvedilol's metabolism 
(CYP2D6). The ranking between the three tested inhibitors obtained in this study, with sertraline being the most potent inhibitor, is consistent with other studies reported in the scientific literature [17].

These data are consistent with other reports regarding the CYP2D6 inhibitory effect of sertraline, fluvoxamine and bupropion in vitro [27-29] and in vivo $[13,30,31]$. Recently, a study that describes the in vitro drug-drug interaction between carvedilol and citalopram was published [20]. The three inhibitors used in this study can be compared with citalopram, based on the same PK parameters evaluated in both studies (metabolization rate, $\left.\mathrm{AUC}_{0-30}\right)$. As a result, sertraline and fluvoxamine are stronger inhibitors than citalopram, while bupropion has a similar potency to citalopram. Furthermore, there are few studies that showed pharmacokinetic drug-drug interactions between carvedilol and CYP2D6 inhibitors, like fluoxetine [32] and paroxetine [33]. Recently, some preclinical studies proving drug-drug interactions between carvedilol and sertraline [34], citalopram [20] and bupropion [35] were published. The in vitro results presented in this study are consistent with the ones resulted from in vivo studies, the inhibitory effect of these antidepressants being therefore proved in both in vitro and in vivo studies.

\section{Conclusion}

The pharmacokinetic drug-drug interactions between carvedilol and three antidepressants frequently used in therapy, i.e. sertraline / fluvoxamine / bupropion were demonstrated in in vitro studies. All the tested antidepressants altered statistically significantly $(p<0.05)$ the in vitro metabolism of carvedilol, modifying the substrate rate of metabolization and the $\mathrm{AUC}_{0-30}$. These results clearly highlight the presence of drug-drug interactions, due to enzymatic inhibition via CYP2D6. Sertraline was proved the most potent inhibitor, followed by fluvoxamine and bupropion. Nevertheless, additional studies on patients should be conducted in order to investigate the clinical relevance of these proved interactions.

\section{Acknowledgement}

This research was supported by Iuliu Hațieganu University of Medicine and Pharmacy, Cluj-Napoca [Internal Research Grant PCD/7690/1/15.04.2016 and PCD/5200/1/1.03.2017].

\section{References}

1. World Health Organization, Cardiovascular diseases (CVDs)

- Fact sheet, 2017. Available from: http://www.who.int/ mediacentre/factsheets/fs317/en/

2. McTavish D, Campoli-Richards D, Sorkin EM. Carvedilol. A review of its pharmacodynamic and pharmacokinetic properties, and therapeutic efficacy. Drugs. 1993;45:232-258.

3. Kilickira-Avci B, Oto A. Preferred beta-blockers for the treatment of heart failure. E-J Cardiol Practice. 2010;5:1-9.

4. Neugebauer G, Akpan W, Kaufmann B, Reiff K. Stereoselective disposition of carvedilol in man after intravenous and oral administration of the racemic compound. Eur J Clin Pharmacol. 1990;38:S108-S111.

5. Neugebauer G, Neubert P. Metabolism of carvedilol in man. Eur J Drug Metab Pharmacokinet. 1991;16:257-260.

6. Oldham HG, Clarke SE. In vitro identification of the human cytochrome P450 enzymes involved in the metabolism of $\mathrm{R}(+)-$ and S(-)-carvedilol. Drug Metab Dispos. 1997;25:970-977.

7. Gehr TW, Tenero DM, Boyle DA, Qian Y, Sica DA, Shusterman NH. The pharmacokinetics of carvedilol and its metabolites after single and multiple dose oral administration in patients with hypertension and renal insufficiency. Eur J Clin Pharmacol. 1999;55:269-277.

8. World Health Organization, Depression - Fact sheet, 2018. Available from: http://www.who.int/news-room/fact-sheets/ detail/depression/

9. Stahl SM, Pradko JF, Haight BR, Modell JG, Rockett CB, Learned-Coughlin S. A review of the neuropharmacology of bupropion, a dual norepinephrine and dopamine reuptake inhibitor. Prim Care Companion J Clin Psychiatry. 2004;6:159-166.

10. Jefferson JW, Pradko JF, Muir KT. Bupropion for major depressive disorder: Pharmacokinetic and formulation considerations. Clin Ther. 2005;27:1685-1695.

11. Maneeton N, Maneeton B, Intaprasert S, Woottiluk P. A systematic review of randomized controlled trials of bupropion versus methylphenidate in the treatment of attention-deficit/ hyperactivity disorder. Neuropsychiatr Dis Treat. 2014;10:14391449.

12. Hare DL, Toukhsati SR, Johansson P, Jaarsma T. Depression and cardiovascular disease: a clinical review. Eur Heart J. 2014;35:1365-1372.

13. Kotlyar M, Brauer LH, Tracy TS, Hatsukami DK, Harris J, Bronars CA, Adson DE. Inhibition of CYP2D6 activity by bupropion. J Clin Psychopharmacol. 2005;25:226-229.

14. Katoh Y, Uchida S, Kawai M, Takei N, Mori N, Kawakami $\mathrm{J}$, et al. Onset of clinical effects and plasma concentration of fluvoxamine in Japanese patients. Biol Pharm Bull. 2013;33:19992002 .

15. MacQueen G, Born L, Steiner M. The selective serotonin reuptake inhibitor sertraline: its profile and use in psychiatric disorders. CNS Drug Rev. 2001;7:1-24.

16. Cascade EF, Kalali AH, Thase ME. Use of antidepressants: expansion beyond depression and anxiety. Psychiatry (Edgmont). 2007; 4:25-28.

17. Spina E, Santoro V, D'Arrigo C. Clinically relevant pharmacokinetic drug interactions with second-generation antidepressants: an update. Clin Ther. 2008;30:1206-1227.

18. Gheldiu AM, Popa DS, Loghin F, Vlase L. Oxidative metabolism of estrone modified by genistein and bisphenol A in rat liver microsomes. Biomed Environ Sci. 2015;28:834-838.

19. Kremers P, Beaune P, Cresteil T, de Graeve J, Columelli S, Leroux JP, Gielen JE. Cytochrome P-450 monooxygenase activities in human and rat liver microsomes. Eur J Biochem. 1981;118:599-606.

20. Abrudan MB, Muntean DM, Popa DS, Gheldiu AM, Neag MA, Vlase L. Inhibitory effect of citalopram on the pharmacokinetics of carvedilol in rats and in vitro models. Pharmacology. 2017; 100:301-307.

21. Cotreau MM, von Moltke LL, Beinfeld MC, Greenblatt DJ. Methodologies to study the induction of rat hepatic and intestinal cytochrome $\mathrm{P} 4503 \mathrm{~A}$ at the mRNA, protein, and catalytic activity 
level. J Pharmacol Toxicol Methods. 2000;43:41-54.

22. Butnariu A, Popa DS, Vlase L, Andreica M, Muntean D, Leucuţa S. New high-throughput liquid chromatographic tandem mass spectrometry assay for therapeutic drug monitoring of carvedilol in children with congestive heart failure. Rev Romana Med Lab 2009;15:7-15.

23. Lynch T, Price A. The effect of cytochrome P450 metabolism on drug response, interactions, and adverse effects. Am Fam Physician. 2007;76:391-396.

24. von Moltke LL, Greenblatt DJ, Schmider J, Wright CE, Harmatz JS, Shader RI. In vitro approaches to predicting drug interactions in vivo. Biochem Pharmacol. 1998;55:113-122.

25. Kremers $P$. In vitro tests for predicting drug-drug interactions: the need for validated procedures. Pharmacol Toxicol. 2002;91:209-217.

26. Frishman WH. Carvedilol. N Engl J Med. 1998;339:17591765 .

27. Crewe HK, Lennard MS, Tucker GT, Woods FR, Haddock RE. The effect of selective serotonin re-uptake inhibitors on cytochrome P4502D6 (CYP2D6) activity in human liver microsome. Br J Clin Pharmacol. 1992;34:262-265.

28. Hesse LM, Venkatakrishnan K, Court MH, von Moltke LL, Duan SX, Shader RI, et al. CYP2B6 mediates the in vitro hydroxylation of bupropion: potential drug interactions with other antidepressants. Drug Metab Dispos. 2000;28:1176-1183.
29. von Moltke LL, Greenblatt DJ, Cotreau-Bibbo MM, Harmatz JS, Shader RI. Inhibitors of alprazolam metabolism in vitro: effect of serotonin-reuptake-inhibitor antidepressants, ketoconazole and quinidine. Br J Clin Pharmacol. 1994;38:23-31.

30. Sproule BA, Otton SV, Cheung SW, Zhong XH, Romach MK, Sellers EM. CYP2D6 inhibition in patients treated with sertraline. J Clin Psychopharmacol. 1997;17:102-106.

31. Todor I, Popa A, Neag M, Muntean D, Bocsan C, Buzoianu A, et al. Evaluation of the potential pharmacokinetic interaction between atomoxetine and fluvoxamine in healthy volunteers. Pharmacology. 2017;99:84-88.

32. Graff DW, Williamson KM, Pieper JA, Carson SW, Adams $\mathrm{KF} \mathrm{Jr}$, Cascio WE, et al. Effect of fluoxetine on carvedilol pharmacokinetics, CYP2D6 activity, and autonomic balance in heart failure patients. J Clin Pharmacol. 2001;41:97-106.

33. Stout SM, Nielsen J, Bleske BE, Shea M, Brook R, Kerber K, et al. The impact of paroxetine coadministration on stereospecific carvedilol pharmacokinetics. J Cardiovasc Pharmacol Ther. 2010;15:373-379.

34. Abrudan MB, Muntean DM, Neag MA, Vlase L, Gheldiu AM. The influence of sertraline on the pharmacokinetics of carvedilol. A preclinical study on rats. Farmacia. 2017;65:557-562.

35. Abrudan MB, Muntean DM, Gheldiu AM, Neag MA, Vlase L. The pharmacokinetic interaction study between carvedilol and bupropion in rats. Pharmacology. 2017;99:139-143. 